\title{
Towards interoperable e-Health system in Tanzania: analysis and evaluation of the current security trends and big data sharing dynamics
}

\author{
Marseline Michael Mtey ${ }^{1 *}$ and Mussa Ally Dida ${ }^{2}$ \\ Research Scholar, Nelson Mandela African Institutions of Science and Technology, Arusha, Tanzania ${ }^{1}$ \\ Lecturer, Nelson Mandela African Institutions of Science and Technology, Arusha, Tanzania ${ }^{2}$
}

Received: 10-September-2019; Revised: 24-October-2019; Accepted: 27-October-2019

(C)2019 Marseline Michael Mtey and Mussa Ally Dida. This is an open access article distributed under the Creative Commons Attribution (CC BY) License, which permits unrestricted use, distribution, and reproduction in any medium, provided the original work is properly cited.

\begin{abstract}
In this paper an insight on various e-health interoperable systems was reviewed to discover strengths and challenges faced during sustainable implementation. It covered local, national and regional coverage of integrated systems towards implementation of a single unified e-health system. Peer reviewed and grey literatures were consulted to discover global and local trend and efforts towards implementations of e-health interoperable systems. The available systems and frameworks from the European Union, Asia, America, Oceania and Africa were analyzed for their strengths and challenges. Various policies, guides as well as free and proprietary standards associated with e-health interoperability was reviewed to understand the common standards adopted by the majority of existing systems. The findings of the analysis are useful for policy makers on the best ways to implement interoperable systems in developing countries by focusing on the existing infrastructures and the environment. Similarly, the strengths and challenges encountered by interoperable systems were also examined to provide recommendations for future studies.
\end{abstract}

\section{Keywords}

Interoperability, Electronic health records, Interoperable frameworks, Big data, Security, Tanzania.

\section{Introduction}

With the evolution of ICT and computing, various industries and business sectors have found better and sophisticated ways of service provisioning through technology integration. Steadily, some old ways of manual paper work are being replaced by upgraded electronic systems or the legacy systems by modern technologies. The integration results to various eservices with great value for service delivery becomes at the fingertips of consumers [1]. Migrating to e-technology also improves and simplifies online information and data sharing process by breaching geographic barriers among individuals [2]. Ecommerce, e-government, e-learning and e-health are few examples of online services that are simplified. One can easily do shopping, reserve tickets, do banking services, and even process legal contracts electronically. Similarly, health services are provided electronically as e-health in which early detection, reporting and response of health-related issues can be done easily [3].

*Author for correspondence
In e-health, patients' diagnosis, prescriptions and monitoring can be done whenever they are with the application of a single or integrated system. According to the World Health Organization (WHO), Information and Communication Technology (ICT) improves health provision by accelerating the data sharing provided that there is a unified standard and protocols to be followed [4,5]. In an information sharing domain, communication can be through Machine-to-Machine (M2M) or any other form of exchanging data through wired or wireless medium with or without human intervention [6,7]. The communication can be within a single site or multiple sites depending on the mode of system integration. Integrated systems can leverage the advantages of being linked together, making data sharing easier. The formed unified or interoperable system has simplified information sharing process since multisite, multi-national and multi-branch organizations can work as a single organization. Various levels exist in interoperability for ensuring that data sharing is possible. They range from technical, semantic and organizational interoperability as shown in Figure 1 [8]. The technical (syntactic) interoperability deals 
with the systems communication, protocols, and the data exchange between the systems [8]. It includes systems' hardware and software parts, and other supporting components required for machine-tomachine communication. Having all necessary infrastructure for communication, the data or contents are to be shared by users on semantic interoperability. It deals with humans' domain in data exchange rather than machine to support information sharing than content interpretation by ensuring meaning extraction from shared data [9]. On the other hand, organizational interoperability focuses on linking organizations together to collaborate in business processes within and outside the organization [10]. The majority of organizations have adopted those interoperability levels by having their local (legacy) systems updated or replaced to listen to one another by following standards like IEEE and HL7 or set of protocols [11].

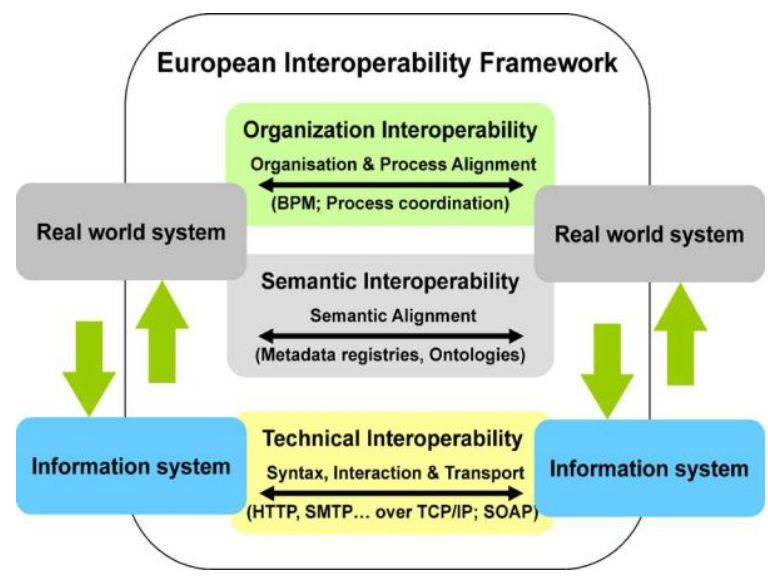

Figure 1 Levels of interoperability according to EU

By integrating the systems, communication barriers in service provision like e-banking and e-health systems is broken since customers can be served anywhere because the information are readily available anywhere and anytime. However, due to the increased number and diversity of the participating systems, there is a high security risk of those nodes (systems and devices) and the communication medium (wired and wireless).

In an e-health particularly, where patients' records are highly confidential, if any, of the systems in interoperability is compromised, security breach of the whole system could happen [12-14]. This is obvious since with any introduction of new device into interoperability, including mobile devices, Internet-of-Things (IoT) Devices, and multiple HIS (Health Information Systems), more security 226 challenges are introduced. That is to say, with each component its own characteristics contribute to the combined challenges associated with the integrated system [15]. Furthermore, some devices, taking part in interoperability have limited storage capacity, so they are forced to outsource their Electronic Health Records (EHRs) or patient health information to third-party service providers or other systems with higher storage power like cloud service providers $[16,17]$.

Outsourcing of these complex patients' health information within health care systems with associated information including demographics, medications, laboratory test results, diagnosis codes, and procedures reliefs the computation burden from those components [18-20]. However, the process may lead to security challenges associated with the device and those associated with the third-party service provider. For the devices, limitations of computing and storage capability are among challenges that call for outsourcing to third party service providers leading to more challenges [21]. In addition, increased number of devices with unclear privacy details, add more complexity to the data and information sharing process [14]. Similarly, there may exist data leakage among the medium of communications between the devices since a man in the middle attack is a common threat $[12,22]$. As a result, various efforts have been taken by different interoperable systems' developers aiming at securing EHR and the associated components.

In this study therefore, various researches on interoperable systems were reviewed, analyzed and evaluated based on their big data sharing dynamics. It included local, national, regional and global efforts towards a unified interoperable system. The aim was to discover strengths and challenges encountered in implementation for the purpose of informing the decision makers on the best approach and procedure in interoperable systems implementation. The next sections cover the review of the guiding standards, some efforts and challenges encountered towards interoperability and finally provide recommendation for policy makers and for further studies.

The study aimed at analyzing various literatures on interoperable systems, based on their big data sharing dynamics. Various standards guiding interoperability and the implementation efforts and observed challenges in different countries, regions and globally were also reviewed. Based on the noted strength and challenges, the authors recommended ways to 
successfully implement an interoperable e-health system to the policy makers.

The next parts of this paper start by a brief explanation of the methodology of this paper followed by discussion of the standards organizations responsible for interoperability. It is followed by analysis of global efforts and challenges towards successful implementation of e-health interoperable systems. Similarly, the observed challenges in big data sharing in those systems are also discussed. Based on the analysis, the authors have provided contribution in the followed section. Finally, recommendations are put forward for further studies and policy makers to successfully implement interoperable e-health systems sharing big data.

\section{Methodology}

This paper adopted documentary review methodology by visiting peer reviewed and grey literatures about the trend of global eHealth interoperability. Documents and reports from indexed and unindexed databases were analyzed to get a clear understanding of the up to date concepts. Those literatures provided an understanding of the standards guiding interoperability, implementation efforts and observed challenges in different countries, regions and globally.

The experience-based design was also used by authors to analyze the documents for effective recommendation for care givers, systems designers and policy makers.

2.1Discussions

2.1.1Standards towards interoperability E-Health systems

According to WHO and International

Telecommunication Union (ITU), e-Health interoperability is the capacity of more than two systems to share and use the data in a meaningful manner [23-25]. Similarly, according to [26] and [27], interoperability is the process of multiple systems to exchange and be capable to use the data. For systems to interoperate, there must be standards, formats and guides that must be followed for them to be able to talk to one another. Those systems must provide common platform for other to simplify the sharing of EHR to promote cooperation among them and improve service provisioning [19]. ITU, Health Level Seven (HL7), International Organization for Standardization (ISO) and its families, Clinical Data Interchange Standards Consortium and European Committee for Standardization (CEN) are some of the common standards organizations with compartments dealing with certain component of interoperability [28, 24,5]. For example, the family of ISO 27000 series is concerned with information security that controls secure information sharing and storage $[29,30]$. ITU on the other hand, is responsible for defining telecommunication networks by setting ITU-Recommendation standards. It foresees all communication mediums by ensuring that those standards are followed. On the other hand, ISO which is the largest standard development organization with more than 168 members is responsible for developing E-health standards through its health informatics technical committee, ISO/TC 215. The standards support the growth in ICT usage in healthcare domain and facilitate secure and seamless exchange of health information to authorized users [31]. Founded in 1987 HL7 oversees comprehensive framework and standards for electronic data exchange [32].

Those standards need to be adopted and implemented to support clinical practice, management, delivery and evaluation of services. Currently, the majority of developed and developing countries have adopted different standards that guide them towards integration of their local systems at national level, towards having a single unified e-health system while trying to mitigate some observed challenges to promote information quality [33]. Their efforts and challenges towards sustainable implementation of the process are described in the subsequent sections.

\section{Analysis of efforts and Success towards implementation and adoption of Interoperable E- Health Systems}

There are some regions and nations that have successfully integrated their legacy and stand-alone systems, though others are working hard towards achieving this. The efforts in developed countries with mature and better network infrastructures are promising compared to the middle and low economy countries.

2.1.2Efforts at European union

For Europe in particular, the efforts started since 2008 when an interoperable exchange of eHealth information framework, the epSOS (Smart Open Services for European Patients) was established to manage Patient records and electronic Prescription (ePrescription) services [34]. In collaboration with the European Committee for Standardization Group of Health Informatics (CEN/TC 251), a standard for patients' record summary was created. Similarly, the European Interoperable Framework (EIF) was 
formed to ensure integration of all member states by adopting some of available standards and deliver public interoperable services. As a framework to public sectors in the European Union (EU), EIF provides guides through European Interoperability Reference Architecture (EU-IRA) to design, implement and maintain interoperable systems [35]. It is a commonly agreed architecture for defining guidelines and common principles, models and recommendations towards interoperability from National, Regions and local levels, embracing public administrators, citizens and businesses. It ensures that all National Interoperable Frameworks (NIFs) are built upon EIF by adding new system components or fine tune the existing system's elements to be able to interoperate. It also provides guidance for the systems that are built for specific tasks like the ehealth systems, following Domain Interoperability Framework (DIF) [36]. Those systems should remain compatible with the EIF to capture its requirements as shown in Figure 2 [37].

By 2017, efforts by some EU members worth an example, since some have shown up-to a high uptake for the NIF including Estonia, Denmark, Finland and Netherland [38]. Cyprus and Austria are among other that have shown more than $94 \%$ effort on the NIF uptake towards successful adoption of EIF. Their efforts in implementing interoperable e-health systems are of high level [39]. However, countries like Belgium, Portugal and Ireland are still struggling to reach $50 \%$ uptake while Italy, Spain and Sweden have $75 \%$ uptake for NIF and are now moving towards regional and international interoperability.

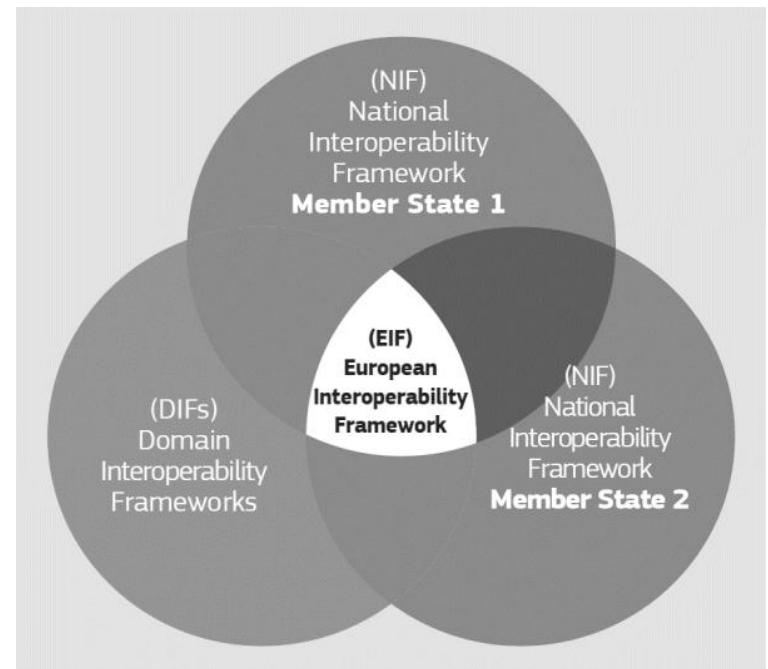

Figure 2 Relationship between EIF, NIFs and DIFs
The efforts have also crossed borders to form a unified EU eHealth system since most European nations have already integrated their HIS. Their efforts are now aiming at having European E-Health Interoperability framework to connect all the integrated systems from member states.

Denmark, Finland and Estonia are among the pioneers of the technology put under the "beacon group" by the European e-health task force report's recommendation. With the 28-member states, the EU digital agenda aims at fostering a harmonious and complementary approach of eHealth through health and ICT policy. They aim at having a long-term plan for creating a harmonized Europe through interoperability of eHealth services since 2015 and enable cross-border exchange of data between member states. However, due to some discrepancies, challenge of health care systems among the member states, health policies and strategies, legislations and progress in national e-health implementation hinders full implementation of the framework [37].

\section{National efforts towards e-health interoperability}

In Denmark, the Danish e-health portal represents implementation where clinical data/information is shared with patients on a national scale. The EU identified Denmark as a country with a great potential, among other European countries in implementation and adoption of e-health [37]. The healthcare system in Denmark is split into state, region and municipal with each level responsible for its own tasks [40]. The municipal level takes care of all community-based services and care, including nurseries, nursing places and rehabilitation centers [37]. In collaboration with regions, it also controls primary and secondary healthcare centers with full authority. On top of that, all public hospitals and their units are owned and managed by regions, though the overall regulator of all functions of the two levels is the government [37]. The government is responsible for maintaining all the legislation and provide overall guidelines about e-health implementation.

Finland on the other hand has an ambitious e-health and e-care strategy to develop national patients' portal functionality. Data exchange with the European e-health systems are made possible through $\mathrm{X}$-Road, which is a data sharing layer for public and private sectors [41]. This mechanism was initially intended for exchange of data between Estonia and Finland to allow the citizens to access to their medical records outside their country when a need 
arises. However, through its implementation, various challenges emerged, including fear of people that electronic communication cannot replace a personal visit to a healthcare. Similarly, it was challenged by having unclear terms of use and inaccessibility of eservices. To overcome those challenges, some unavailable functions during the initial implementation like monitoring and measurement review or renewal of prescription, access to medical test results and safe communication are stated in the new e-health strategy to be implemented by 2020 [42].

In Italy, the Government has set an Essential Level Care (LEA) through the Italian National Health System (NHS) [43]. With a national framework called National Healthcare Information System (NSIS), unified EHRs are defined and regulated at a national level [34]. It ensures that, the health care system managed by regions, must meet the national requirements. The regional governments are responsible for pursuing the national strategy and operational objectives at local level as well as control of their own functions. Through this, a harmonious ehealth policy's definition will be influenced by respecting the regional autonomy hence successful national e-health system [43].

In 2015 all autonomous provinces and regions were required to implement EHR or "Fasciolo Sanitario Elettronico (FSE)" and provide it for use in healthcare as well as research study, planning, management, monitoring and assessment in healthcare. To add credibility to this, explicit provision was provided for FSE to be compliant with any legislation on privacy and processing of personal sensitive data. Furthermore, the provision sets a national infrastructure to ensure FSE/EHR interoperability at both national and European levels. Few encountered challenges during implementation include unavailable E-health policy at a national level and a large degree of autonomy by the regions with mandate to decide what and how to invest in technology or any other business.

Not left behind, the Netherlands observed privacy risks, information overload and liability issues as challenges that are to be worked out towards successful implementation of the national framework and EIF [27]. General practitioners, nurse practitioners, physiotherapists, and doctor assistants also noted other challenges, including technology illiterate, technology failure, costs and overall security. All of them described those challenges by claiming technology illiteracy as an outstanding challenge among others [27].

Furthermore, the United Kingdom has put in place the National Health Service (NHS) which provide patient record summary since 2008 [44, 45]. This system produces a pdf summary care record which is stored in NHS spine [46]. The patient can access their EHR in the form of the summary care record nationally wherever they are. Since 2015 all the Healthcare providers were required to opt from available e-Health Systems but must adhere to SNOMED CT (Systematized Nomenclature of Medicine Clinical Terms) coding system [47]. SNOMED CT the popular global clinical terms adopted by most clinical sectors [48].

Estonia is one of the EU countries that has successfully implanted National interoperable ehealth systems [49]. It has a majority of the local healthcare providers with e-health systems integrated to the national interoperable e-health system. Data security management in Estonia is puta as a strategic function of any organization to ensure the safety of EHR. The heads of those organizations must ensure that this responsibility is not delegated to IT department due to its sensitivity. Every healthcare provider in Estonia is responsible for ensuring that the neighbor health care provider does not fail in data protection since a failure in one system means failure of the whole interoperable system. Furthermore, all national cyber security processes are put under the Estonian Information System Authority (EISA) which also coordinate the national information system and all ICT related issues of the state including data communication and information security [41]. It adopts three-tiered reference security system to be used by the state and all local government and private agencies engaging in public services.

Estonia as a member of the EU, limits the amount of data shared with other states due to various security risks, yet to be cleared by to ensure that the citizens have secure access to their EHR. Citizens inside or outside the country can use their national ID card for authentication to access their EHR in the e-Health Patient Portal [50]. With the adoption of HL7 and DICOM message standards, EISA also uses the government X-Road middleware software for security during data transport and sharing processes. To further ensure secure and private exchange of information, it replaced the hash chaining cryptographic mechanism by a blockchain 
technology [51]. Other aims of migrating to blockchain was to provide proof of database record integrity and to tamper proof activity $\log$ s so that system configuration and activity $\operatorname{logs}$ are not manipulated after the agreed correct state.

Generally, there are various challenges that EU states encountered towards EIF implementation. Among them is the availability of varying regulatory framework and national healthcare system structures in individual states and countries [34]. Also, some countries do not have a NIF or there exist no institutional interoperable framework. Similarly, other countries have no facility level framework leading to difficulties in forming a regional and later a national level framework [37]. In addition, there exist no balance between governmental and nongovernmental sectors calling for a need to have a unified guideline for successful implementation [52]. From those efforts by individual states therefore, since January 2019 the European Patient Summary for Unplanned, Cross-border Care was approved, in partnership with HL7 as starting point for the European Guidelines on cross-border care that emerged from the epSOS [34]. The aim was to enable people to access and share their health information for emergency or unplanned care across Europe.

\subsection{Efforts in Asian countries and oceania}

In Asia specifically the Southern Asia, Malaysia and India endorse the use of open standards that can help achieve better interoperability quickly to reduce the cost of implementation and ensure ownership of the final deployed system [53]. The Indian National eHealth Authority (NEHA) was given the mandate to foresee the adoption and use of ICT in all healthcare providers and eventually a national unified single system. It was a requirement that even a smallest Primary Healthcare Centers (PHCs) with a single doctor or a specialty healthcare with complex services that serves more than 1.24 Indians should be connected to simplify service provisioning [54]. This integration was to ensure that the diverse Health Information Systems (HIS) developed by public and private sectors serve patients well with the agreed and set national standard, eventually simplifying the government's task of reporting and monitoring what is happening in India healthcare system [55].

Currently the new National Health Policy 2017 is adopted throughout the country encouraging cooperation and participation between the stakeholders [56]. It emphasizes on mainstreaming of multiple available systems to allow patients to have access and informed choice while inspiring cross referrals across these systems [57]. Therefore, to accelerate integration and encourage standardization and exchange of data, India formulated an EHR standard and became the member of International Health Terminology Standards Development Organization (IHTSDO) since 2014 agreeing to the use of SNOMED-CT by all healthcare service providers [58]. This was catalyzed by the adoption of EHR/EMR for data capture, storage, view, presentation, and transmission to achieve syntactic and semantic interoperability of health records [59]. In Australia which is an oceanic country, control of National eHealth Transition Authority (NEHTA) due to some noticed legal and political complications [60]. NEHTA was established by the Australian, State and Territory governments to help in enabling sustainable e-health service provision especially in interoperability. It is also responsible for developing better ways of collecting and secure sharing of electronic health records [61]. Following the principles included in The Open Group Architectural Framework (TOGAF) which describes ways to develop customized enterprise architecture for any organization, a Service-Oriented Architecture (SOA) for Australia was developed. This architecture was a result of a need to gain competitive advantage by managing applications interacting in interoperable systems [62].

To manage secure sharing of data, a Personally Controlled Electronic Health Record (PCEHR) was later established to ensure secure sharing of information among healthcare providers. It consists of an Individual Healthcare Identifier (IHI) issued to users for secure controlling of information [61]. In order to improve interoperability, various improvements were done to NEHTA to produce its second version known as IF2 intended to improve conversation between stakeholders when dealing with e-health systems [63].

From 1 July 2012 PCEHR was renamed to My Health Record System (MyHRS) which contains individual's health information, including prescriptions, any treatments or allergies [64]. Though MyHRS facilitates better communication between various healthcare providers, it has some pitfalls which are being worked on including illiteracy level of users [65]. In addition, there are resistance from some health practitioners and unknown future consumers realization of the system [66]. 
3.2Efforts in Africa and America

In Africa, the adoption of interoperability framework is still under research for proper standards to be adopted. Some countries have jointly developed their integrated systems to facilitate data sharing though do not cover the whole continent. Ethiopia, Kenya, Malawi and South Africa are currently validating a health system to support HL7 base data sharing between Electronic Medical Records and (District Health Information System, Version 2) DHIS2 which is an open source program used by more than 60 countries [67]. The first version was first developed as a web-based, open source software in South Africa in 1996, and is being upgraded by various projects running in some countries of Asia, Africa and the United States though the Health Information Systems Programme (HISP) coordinated by University of Oslo in Norway [68].Other efforts are towards improving internal health systems to be able to share EHRs based on EA framework to help multiple systems interoperability [69]. From the author's knowledge, there is still few published researches on the progress towards a unified eHealth system. There is also limited coordination especially at national level due to majority of projects being donor driven resulting to rigidity in revealing their system's infrastructure. Majority of those projects are available at a single facility with no option or possibility to scale-up and not capable of exchanging healthcare data. Some nations in Africa adopted low level ehealth standards that do not support sharing of crucial healthcare information necessary to support continuity of care [70].

Though number of standards developed by Standards Developments Organizations (SDOs) lead to low pace of adoption of e-health, they also do not define a unified area. Therefore, with the availability of conflicting and overlapping standards, difficulty in combining standards from different SDOs, and high cost of converting to new standard-based solution hinders the quick uptake of interoperable e-health at national level and eventually at regional level. However, some nations have moved a step ahead in successfully putting forwards various bodies and guides to coordinate implementation of e-health.

In Rwanda for example, the implementation started with a web based HIMS, The Rwanda HIMS (RHMIS) since 2012 by collecting data from local systems to DHIS2 based systems [71]. This R-HMIS has improved data management tools to improve the way Rwandans' are served by healthcare service providers. The existed parallel systems that were running like Electronic Integrated Disease Surveillance and Reporting (eIDSR), HIV and the TB systems were migrated and integrated into DHIS2 [72].

Mauritius has been a front runner in national ICT policy and liberalized the National telecommunication framework since 1989 though they lag behind in implementation of e-health systems [73]. Lack of proper e-health infrastructure caused by limited awareness of benefits of e-health by policy makers, health authorities and health practitioners are among the challenges that the country faces [73]. In addition, there is neither a national enabling policy environment nor national regulatory framework for e-health. Furthermore, healthcare stakeholders miss collaboration among themselves due to weak leadership and inadequate human capacity to plan and apply e-health solutions. With those challenges, there is however some efforts on the other side of integrating and using mobile devices application in provision of healthcare, referred to as mhealth [74]. Mhealth in Mauritius is on the raise with numerous numbers of free mobile application available to be downloaded for free in google application store [73]. Among them is the Doctor Assistant which is a free EMR already adopted by the Mauritius Research Council and State Informatics Limited to be used as a guide towards development of National Healthcare Information System. This system is to be used to facilitate the drive towards having e-health system that will facilitate timely and secure access to EMR including big data and open data that will be useful in various medical purposes when data mining is done. On the other hand [75] has recently designed a framework for the Implementation of eHealth as starting point for the country towards a country interoperable eHealth system.

In the Republic of South Africa, the National Health Insurance (NHI) policy is responsible to outline ways to establish an effective strategy for national e-health system. The established 2012-2016 e-health strategy outline ways to resolve implementation of effective and efficient South African e-Health system that can produce real time information for decision making. In this strategy healthcare informatics, m-health, eprescription, EMRs, and telemedicine are included. The strategy has different key compartments to ensure proper implementation of the policy [76]. Some of the components include stakeholders' engagement to understand the local system in place during the integration process. This will lead to 
understanding of whether the system need to be customized to meet the national requirements or the national system need to be customized to meet the local requirements. Standards and interoperability are other components governing systems interoperability, by providing common way of messaging ensures understanding between the systems. Similarly, Governance and regulation were observed to be important driving force for sustainable existence of the system they ensure compliance with the set regulations. Furthermore, other strategic points that were set include e-health foundation, tools and application to support healthcare delivery, and monitoring and evaluation.

From the abandon list of e-health standard setting organizations, South Africa has adopted ISO 21549 in addition to its coordinated local standard referred as SANS 828-2 [77]. SANS 828-2 Health Informatics-Health Smart (HS) card and private Healthcare Information Standard Committee (PHISC) help to maximize cooperation in e-health standards across the country. They help to successfully implement eHealth strategy, technical standards and ensure national and international compatibility, interoperability, open architecture, modularity and capacity for upgrade of the legacy systems [78]; [79]. The country is hoping to achieve e-health implementation technology to improve healthcare service delivery by building on already existing systems in private and public sectors by filling the observed gap. However, there are some encountered challenges to a successful implementation including network access bandwidth, staff training, formulation and implementation of new management, and record that were to be resolved before implementation. Similarly, formulation of a National Master Patient Index (MPI) for the patients' record was to be in place while observed security challenges including location security, data transmission security and data storage security. These and other challenges are addressed by National Health Normative Standards Framework (HNSF) which is a generic e-health Architectural component to ensure all forms of interoperability in SA including semantic, syntactic, organizational and technical.

Enterprise Architecture (EA) framework is also adopted for managing and aligning organizations assets especially ICT, people, operations, and projects with operational characteristics. In ICT perspective, it defines how this technology should be used to support a healthcare business. The HNSF can be fed into the EA for the national healthcare system to improve record sharing process.

In Kenya, various projects concerning e-health focusing on $\mathrm{m}$-health application in primary care are implemented. They concentrate on HIV/AIDS though most of them are not evaluated though the National vision 2030 policy set by the government identify ICT as a key determinant in attaining an economic pillar relating to business process outsourcing. The focus areas include telemedicine, health information system (EHR), m-health, e-learning and health information for citizens.

To emphasize and strengthen the focus on e-health, the national e-health strategy 2011-2017 addressed challenges that could hinder proper utilization of ICT to healthcare. The existence of multiple e-health projects funded by development agencies and international non-governmental organizations, hinders sustainable implementation of e-health systems. Similarly, ownership of the systems and fragmentation of them causes another challenge towards integration. Furthermore, due to unavailability of a national e-health standard and regulatory framework to guide in interoperability, the available fragmented systems cannot be integrated with the national health information system [80].

For Uganda, the Ministry of Health $(\mathrm{MOH})$ initiated a project in collaboration with the Uganda Communication Commission (UCC) called ICT4MPOWER to strengthen the healthcare systems information flow from community to national level [81]. The 2013 Uganda National e-health policy stipulated that most e-health applications and products were run in silos and not interoperable or compatible, therefore become difficult to share information and services. In addition, it was noted that several of them have remained as pilot projects for life with no possibility of interoperability due to availability of divergent platforms [81].

In North America, Canada and USA are the pioneers of ehealth systems interoperability. In USA patients use a portal to access their medical records whenever they require to do so. This portal allow intercommunication between healthcare domain and between social care. To further integrate portals, interoperability problems and controlled access within this, new paradigm of trust and security is needed. 


\section{The Tanzanian context}

In Tanzania, the effort is witnessed through various studies and government directives towards integrated Health Information System (iHIS). According to the e-health strategy, iHIS is a collection of integrated (loosely coupled or tightly coupled) standards-based information system that support operation management, and decision making in the health sector. Local systems in the country are integrated with the DHIS2 to support the effort of Ministry of Health to solve the most troubling health issues. Good examples of systems integrated with DHIS2 include eIDSR tool that also uses USSD technology to detect and respond to infectious diseases. The Electronic Logistics Management Information System (eLMIS) is also integrated with DHIS2, to compare and triangulates the service delivery data. According to the 2012-2018 national e-health strategy, a national e-health steering committee (NeHSC) is responsible for ensuring timely implementation of e-health initiatives [82]. Among the strategic goals is to enable health sectors to operate more effectively as connected systems, overcoming fragmentation and duplication of service delivery [83]. It also ensures safe care and timely availability of information when and where needed and remote access to healthcare services for patients in remote rural and disadvantaged communities. Furthermore, support for multi-way communication and sharing of information among clinicians, patients, and care givers within healthcare sector and across partner agencies are other efforts that committee seek to accomplish.

With the adoption of the EA, the Ministry of Health, Community Development, Gender, Elderly and Children (MoHCDEC) and other healthcare stakeholders ensure that this framework will guide in development and implementation of National iHIS. The ministry is ambitious in completing the Tanzania Health Enterprise Architecture (THEA) to guide in development of national integrated HIS [83]. Currently, the framework has been used to design a HIS called Electronic Facility Management System (eFMS), which will be implemented in majority government hospitals in Tanzania in phases [84]. There are two phases towards implementation of the HIS with Phase 0 aiming at developing Hospital Management Information Systems (HoMIS), warehouse for data, and National Health Insurance Fund (NHIF). Phase 1 on the other hand is implemented after successful implementation of Phase 0, enabling HOMIS to support financial, medical, and Human Resource (HR) systems.
Furthermore, the second phase is responsible for implementation of health information mediator and integration of existing information systems. The mediator is responsible for integrating the available local health information systems by ensuring that healthcare providers nationwide adhere to the same standard and guide [85] developed an architecture for data exchange component (DEC) that integrate a single mobile application with multiple eHRs to enhance interoperability. The systems took the advantage of widespread ownership of mobile phones by a large world population. With those efforts, still there is no national unified system in place for interoperability. Since syntactic and semantic interoperability aim at interpretation and meaning extraction respectively, all the efforts aimed at meeting those goals. Interpretation of exchanged data by the other party to aim at extracting the intended information.

4.1Data sharing in interoperable e-health systems The concept of interoperability in e-health systems and components intended for information sharing is accompanied with processing of big data. With a wider advancement from functional (technical) interoperability for data exchange all the way to semantic interoperability, where the exchanged data can be interpreted by each individual system, their benefits in healthcare are clear. According to the survey by [86] more than $75 \%$ of global e-health systems have advanced from technical, semantic and organizational interoperability as indicated by Figure 3 [86]. Thus, data sharing and information extraction are common practice for those systems since they have their structures fine-tuned for interoperability hence able to accommodate data sharing through semantic interoperability.

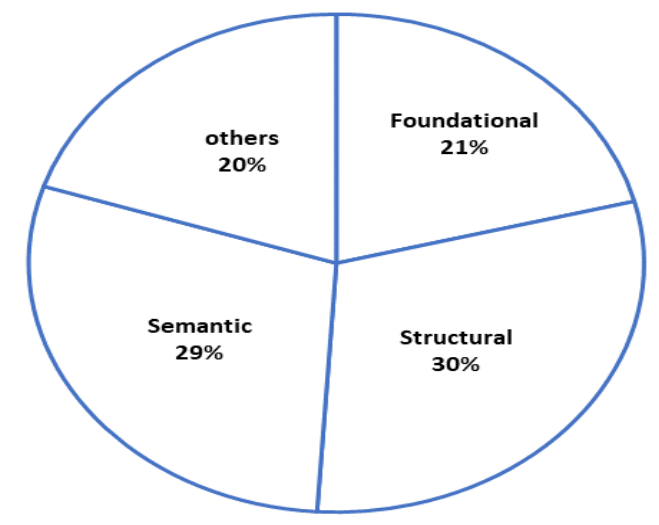

Figure 3 Percentage share of levels of interoperability 
There might exist various challenges that each individual system with interoperability possesses specifically during information sharing and outsourcing [87]. Since an individual e-health system processes large amount of data, integration of more components like IoT and mobile devices increase the size of data hence complexity. The big data (data with high volume, velocity and different variety) may provide useful information when analytics are done [88]. Better diagnosis, decision-making with automated algorithms, data transparency and the patients risk reduction are the result of big data mining [89]. It therefore provides opportunities to improve performance of patient care provisioning and is useful in clinical practices and research specifically in clinical predictions [90]; [91]. Therefore, to enjoy those advantageous features there is a need for special management procedures for big data in healthcare. In Europe, for example, the EU data policy addresses the issue of big data integration in healthcare by initiating data centers and ensuring information confidentiality and security. The policy also provides a means of implementing e-health and m-health and genomics and bioinformatics management. Various ethical and technical challenges to successive sharing of big data in healthcare were observed, including confidentiality and data security as well as access to information since it is clear that PHR should be kept secure regardless of the size. For technical challenges, data reliability, interoperability, management and governance hinder the integration process. Furthermore, the absence of comprehensive health and research policy strategy for big data regardless of initiative by the 2020 European digital agenda for innovation and economic growth throughout the union [92]. Successful implementation of the agenda is a step towards integration of big data to healthcare which may become useful in various predictions and research purposes by whoever adopt it.

\section{Observed challenges in systems interoperability and big data sharing}

Through the literature, it was revealed that there are various challenges that need to be considered if a successful implementation of an integrated e-health system is needed. The availability of multiple standard setting organizations, multiple components to interoperability, device capability, differences in storage systems' architectures, and untrustworthy communication channels are some of the noted challenges. Based on the reviewed studies, some states, especially developed ones experience less challenges in comparison with the developed ones as shown in Table 1.

\subsection{Based on adopted standards}

In systems interoperability, especially e-health, standards to guide the associated fragments is a must since proper messaging formats need to be set and agreed upon by all. However, since those devices are free to choose their own standards setting organization during system implementation, it becomes difficult to have a single harmonized standard for information sharing in interoperable system. Sometimes it requires a component in the interoperability to adhere to local standards and diverse number of regulatory agencies until it becomes fully effective [93]. This makes it difficult to have the components ready made for integration as a plug and play without some modifications especially to the off-the-shelf systems and components. It was noted by [94] that since developing countries focus on adopting open source standards, the unavailability of ready-to-adopt standards and existing barriers to the adoption are the main challenges. The authors added that much efforts focus on adoption and development of standards rather than focusing on information sharing. On the other hand, local standards are set purposely to fit to one's requirement without much considering the future need for integration. Furthermore, the lack of skills and expertise in the field of e-health standardization is another challenge to the integration. For donor funded or government e-health projects, there is a limited guideline on which standards to use and sometimes donors may control the choice [95].

\subsection{Based on devices' computing and storage capability}

Local, national and international systems have different security challenges specifically due to the size of the participating components. Since interoperability is associated with multiple devices with different standards, bandwidth requirements, computing and storage capacity, and make, it is similarly complex to manage them. For example, it requires common standards to enable cooperation among the internet of things devices, taking part in an interoperability since they have different features [96]. Similarly, with either internal, external or hybrid mode of information storage, various challenges may arise among devices. If an internal server is chosen for data storage, malicious internal users may intentionally or unintentionally interlude with the data security. Furthermore, outsourcing 
operation brings more challenges, including the adopted challenges from that third-party service provider, including their curious habit of examining and hence risk of disclosure of the information shared through them. However, if a hybrid mode is chosen, internal and external storage challenges may arise if less precautions are taken.

\subsection{Security challenges}

Security challenges are among the challenges that the majority of systems globally face regardless of efforts. The success of integrated e-health portal in the USA for example, is faced with challenges of controlling patients' access to EMR due to absence of guides for monitoring the process. Furthermore, the availability of numerous systems and components for interoperability brought a new threat about trust of those components especially in security of shared data. Similarly, Denmark faces the same challenge of absence of monitoring access and security of communication media between integrated systems due to integrated e-health systems. This challenge was observed also during system implementation in South Africa. They noted security threat in data transmission in communication medium as well as location and storage security. The Danish government expects to solve these challenges by 2020 while South African recommended the use of local set standard, local architecture and encourage stakeholders' engagement while resolving observed challenges before implementation. Maintaining privacy risks on EHR is another challenge noted during implementation of interoperable systems in the Netherlands. It was suggested that patients' records are sensitive, therefore they are supposed to be kept secure. Since EMR can also be used for research purposes, it was noted by the Estonian government that the security of the data used for research is of vital importance.

Table 1 Common global interoperability challenges

\begin{tabular}{|c|c|c|c|}
\hline $\begin{array}{l}\text { Interoperability } \\
\text { Challenges }\end{array}$ & $\begin{array}{l}\text { Developed } \\
\text { Countries }\end{array}$ & $\begin{array}{l}\text { Low- and Middle-Income } \\
\text { Countries (LMICs) }\end{array}$ & Description \\
\hline Standards & & & Availability of multiple standards \\
\hline Security & & & Devices and Communication security \\
\hline Memory & & & Limited Device memory capacity \\
\hline Bandwidth & & & Network fluctuation \\
\hline Computation & & & Limited devices' computing power \\
\hline Lack of Strategies & & & No guiding policies \\
\hline $\begin{array}{l}\text { Donor Funded Projects } \\
\text { cease }\end{array}$ & & & $\begin{array}{l}\text { Completion of some health supporting } \\
\text { projects }\end{array}$ \\
\hline Systems fragmentation & & & $\begin{array}{l}\text { Diversity of the available systems from } \\
\text { different vendors }\end{array}$ \\
\hline $\begin{array}{l}\text { Lack of guiding } \\
\text { Infrastructure }\end{array}$ & & & No friendly infrastructure \\
\hline
\end{tabular}

\subsection{0ther challenges}

There are other challenges that hinder the efforts of ehealth systems interoperability, including technological illiteracy, unavailability of computers and outdated technology. Also, network fluctuation hampers the efforts to successful interoperable systems. When the network becomes low the interoperability sustainability is affected hindering the shareability of data and information among systems [97]. These challenges also hinder the efforts towards successful implementation of interoperable 235 systems. It respectively requires modern technology and skilled personnel to install and understand or operate the individual or interoperable systems.

\section{Conclusions and recommendations}

Integration of e-health systems is very important in improving healthcare service provisioning due to simplification and ubiquitous access to EMR. Globally, there are various nations and states that have successfully implemented local and national 
integrated systems and are moving towards international interoperability. A good example is the European Union with their EIF a framework for interoperability.

However, since the interoperability is associated with integration of different systems and components, the volume of data being shared become big leading to more complexity. Furthermore, the security of the communication medium, storage location and data used for research purposes must be highly taken into consideration.

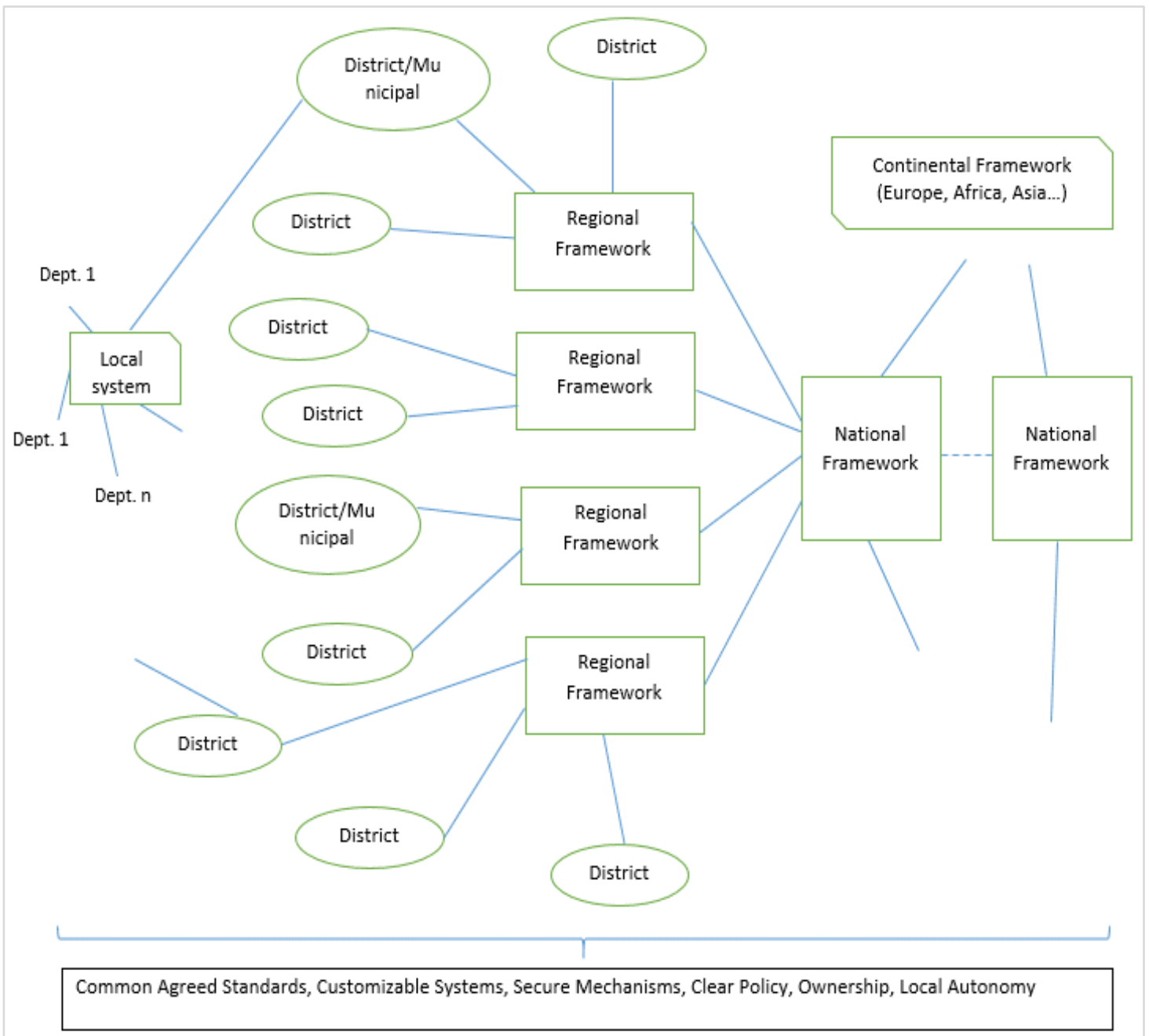

Figure 4 Recommended interoperable e-health system based in Italy and Denmark frameworks

For successful implementation of a national integrated e-health system, there must be issues to be put in place as indicated by Figure 4. Firstly, efforts must be employed to ensure that the local e-health systems at facility level are in good shape. This includes an awareness of the standards being followed, its architectural make-up, means of data storage, and whether the system is capable of integration. Secondly, a regulatory authority must be enacted and clear policies for data sharing, security and privacy of patients' EHR must be maintained since they contain sensitive information. Thirdly, a common standard should be adopted by all the systems, especially messaging standards for the exchanged data to be meaningful and useful to the receiving party.

In addition, all the donor funded projects are supposed to put clear their systems to sustain the process rather than keeping them secret. Furthermore, the use of open standards can help achieve better interoperability than the use of proprietary ones. 
Finally, with successful implementation of local ehealth systems ready for integration, the local system autonomy must be relinquished so that whenever system update is to be done, there will be no challenge.

\section{Acknowledgment}

None.

\section{Conflicts of interest}

The authors have no conflicts of interest to declare.

\section{References}

[1] Owais ST, Khanna S, Mani RS. Building multichannel e-service delivery platform: opportunities and challenges. In proceedings of the special collection on egovernment innovations in India 2017 (pp. 58-63). ACM.

[2] Munyoka W, Maharaj M. Towards the harmonisation of information and communication technology policy frameworks in the Southern African development community. In international conference on information technology systems and innovation 2017 (pp. 1-8). IEEE.

[3] Simon E, Mushi A. eHealth as the trigger initiative that may foster development in health care delivery in Tanzania. In international conference on sustainable ICT, education, and learning 2019 (pp. 39-44). Springer, Cham.

[4] Sylva P, Abeysinghe B, James C, Jayatilake A, Lunuwila S, Sanath D, et al. A review of eHealth policies that underpin global health care digitization. Sri Lanka Journal of Bio-Medical Informatics. 2011; 2(4):118-29.

[5] Ouhbi S, Fernández-Alemán JL, Carrillo-de-Gea JM, Toval A, Idri A. E-health internationalization requirements for audit purposes. Computer Methods and Programs in Biomedicine. 2017; 144:49-60.

[6] Tuna G, Kogias DG, Gungor VC, Gezer C, Taşkın E, Ayday E. A survey on information security threats and solutions for machine to machine (M2M) communications. Journal of Parallel and Distributed Computing. 2017; 109:142-54.

[7] Borgia E. The internet of things vision: key features, applications and open issues. Computer Communications. 2014; 54:1-31.

[8] Vernadat FB. Technical, semantic and organizational issues of enterprise interoperability and networking. Annual Reviews in Control. 2010; 34(1):139-44.

[9] Boateng R, Acheampong B, Bedi I. Preliminary insights into dominant issues, theories and methodologies in platform interoperability research. Twenty-fifth americas conference on information systems, cancun, 2019 (pp. 1-10).

[10] Margariti V, Stamati T. Organizational interoperability in e-government: $\alpha$ case study from the Greek public sector. EGOV-CeDEM-ePart 2018.

[11] Rac-Albu EM, Ciobanu V, Rac-Albu M, Popescu N. Interoperability of medical data through e-Health service in Romania. In international conference on exploring services science 2016 (pp. 683-92). Springer, Cham.

[12] Chokshi A, Patel S. Internet of things (IoT), IoT architecture, security challenges in IoT \& Role of IoT in healthcare industry. International Journal of Engineering Technology Science and Research. 2017; 4(10):822-5.

[13] Bandyopadhyay D, Sen J. Internet of things: applications and challenges in technology and standardization. Wireless Personal Communications. 2011; 58(1):49-69.

[14] Lee I, Lee K. The internet of things (IoT): applications, investments, and challenges for enterprises. Business Horizons. 2015; 58(4):431-40.

[15] Rahmani AM, Gia TN, Negash B, Anzanpour A, Azimi I, Jiang M, et al. Exploiting smart e-Health gateways at the edge of healthcare internet-of-things: a fog computing approach. Future Generation Computer Systems. 2018; 78:641-58.

[16] Li J, Zhang Y, Chen X, Xiang Y. Secure attributebased data sharing for resource-limited users in cloud computing. Computers \& Security. 2018; 72:1-12.

[17] Fernandez F, Pallis GC. Opportunities and challenges of the internet of things for healthcare: systems engineering perspective. In 4th international conference on wireless mobile communication and healthcare-transforming healthcare through innovations in mobile and wireless technologies (MOBIHEALTH) 2014 (pp. 263-6). IEEE.

[18] Black AD, Car J, Pagliari C, Anandan C, Cresswell K, Bokun $\mathrm{T}$, et al. The impact of eHealth on the quality and safety of health care: a systematic overview. PLoS Medicine. 2011; 8(1):1-16.

[19] Stephanie FL, Sharma RS. Health on a cloud: modeling digital flows in an e-health ecosystem. Journal of Advances in Management Sciences \& Information Systems. 2016; 2:1-20.

[20] Yadav P, Steinbach M, Kumar V, Simon G. Mining electronic health records: a survey. arXiv preprint arXiv:1702.03222. 2017.

[21] Pasupuleti SK, Ramalingam S, Buyya R. An efficient and secure privacy-preserving approach for outsourced data of resource constrained mobile devices in cloud computing. Journal of Network and Computer Applications. 2016; 64:12-22.

[22] Kumar P, Lee HJ. Security issues in healthcare applications using wireless medical sensor networks: a survey. Sensors. 2012; 12(1):55-91.

[23] Juma K, Nahason M, Apollo W, Gregory W, Patrick O. Current status of e-health in Kenya and emerging global research trends 1 . International Journal of Information and Communication Technology Research. 2012; 2(1):50-4.

[24] Chituc CM. XML interoperability standards for seamless communication: an analysis of industryneutral and domain-specific initiatives. Computers in Industry. 2017; 92:118-36.

[25] Reis ZS, Maia TA, Marcolino MS, Becerra-Posada F, Novillo-Ortiz D, Ribeiro AL. Is there evidence of cost 
benefits of electronic medical records, standards, or interoperability in hospital information systems? overview of systematic reviews. JMIR Medical Informatics. 2017; 5(3):1-13.

[26] Rezaei R, Chiew TK, Lee SP, Aliee ZS. Interoperability evaluation models: a systematic review. Computers in Industry. 2014; 65(1):1-23.

[27] Nijeweme-d'Hollosy WO, Van Velsen L, Huygens M, Hermens H. Requirements for and barriers towards interoperable eHealth technology in primary care. IEEE Internet Computing. 2015; 19(4):10-9.

[28] Huser V, Sastry C, Breymaier M, Idriss A, Cimino JJ. Standardizing data exchange for clinical research protocols and case report forms: an assessment of the suitability of the clinical data interchange standards consortium (CDISC) operational data model (ODM). Journal of Biomedical Informatics. 2015; 57:88-99.

[29] Evans L. Protecting information assets using ISO/IEC security standards. Information Management. 2016; 50(6):28-33.

[30] https://www.iso.org/obp/ui/\#iso:std:iso-iec:27000:ed5:v1:en:en. Accessed 15 March 2018.

[31] Dolin RH, Rogers B, Jaffe C. Health level seven interoperability strategy: big data, incrementally structured. Methods of Information in Medicine. 2015; 54(01):75-82.

[32] http://www.hl7.org/ . Accessed 15 March 2018.

[33] Bhalla S, Sachdeva S, Batra S. Semantic interoperability in electronic health record databases: standards, architecture and e-health systems. In international conference on big data analytics 2017 (pp. 235-42). Springer, Cham.

[34] Nalin M, Baroni I, Faiella G, Romano M, Matrisciano F, Gelenbe E, et al. The European cross-border health data exchange roadmap: case study in the Italian setting. Journal of Biomedical Informatics. 2019.

[35] Mondorf A, Wimmer M. Contextual components of an enterprise architecture framework for panEuropean egovernment services. Proceedings of the 50th hawaii international conference on system sciences. 2017 (pp. 2933-42).

[36] Kouroubali A, Katehakis DG. The new European interoperability framework as a facilitator of digital transformation for citizen empowerment. Journal of Biomedical Informatics. 2019.

[37] https://www.eesc.europa.eu/en/our-work/opinionsinformation-reports/opinions/europeaninteroperability-framework-implementation-strategycommunication. Accessed 15 March 2018.

[38] Kalogirou V, Charalabidis Y. The european union landscape on interoperability standardisation: status of european and national interoperability frameworks. In enterprise interoperability VIII 2019 (pp. 359-68). Springer, Cham.

[39] Voutsidou S, Moraitis E, Sissouras A, Jelastopulu E, Charalampous G. E-health and primary health care: telemedicine in the greek national health system. International Journal of Recent Scientific Research. 2019; 10(8):34320-5.
[40] Høstgaard AM, Bertelsen P, Nøhr C. Constructive eHealth evaluation: lessons from evaluation of EHR development in 4 danish hospitals. BMC Medical Informatics and Decision Making. 2017; 17(45):1-15.

[41] Paide K, Pappel I, Vainsalu H, Draheim D. On the systematic exploitation of the Estonian data exchange layer X-road for strengthening public-private partnerships. In proceedings of the 11th international conference on theory and practice of electronic governance 2018 (pp. 34-41). ACM.

[42] http://julkaisut.valtioneuvosto.fi/handle/10024/74459. Accessed 15 March 2018.

[43] Domenichiello M. State of the art in adoption of ehealth services in Italy in the context of European union e-government strategies. Procedia Economics and Finance. 2015; 23:1110-8.

[44] Waterson P. Health information technology and sociotechnical systems: a progress report on recent developments within the UK national health service (NHS). Applied Ergonomics. 2014; 45(2):150-61.

[45] Gibson D, Smith L. How to use the summary care record in community pharmacy. PharmaceuticalJournal. 2016.

[46] Adewunmi A. A critical appraisal of the summary care record (SRC) scheme in England: medway maritime hospital case study. University of Sheffield South Yorkshire. 2014.

[47] Hayward-Giles S, Millar J. UK approach to developing SNOMED CT subsets for physiotherapists to use as part of the electronic health record. Physiotherapy. 2015; 101(1):550-1.

[48] Bodenreider O, Cornet R, Vreeman DJ. Recent developments in clinical terminologies-SNOMED CT, LOINC, and RxNorm. Yearbook of Medical Informatics. 2018; 27(01):129-39.

[49] Schiza EC, Kyprianou TC, Petkov N, Schizas CN. Proposal for an e health based ecosystem serving national healthcare. IEEE Journal of Biomedical and Health Informatics. 2018; 23(3):1346-57.

[50] Nøhr C, Parv L, Kink P, Cummings E, Almond H, Nørgaard JR, et al. Nationwide citizen access to their health data: analysing and comparing experiences in Denmark, Estonia and Australia. BMC Health Services Research. 2017; 17(534):1-11.

[51] Efendi S, Siregar B, Pranoto H. Concept designs of patient information security using e-health sensor shield platform on blockchain infrastructure. In proceedings of MICoMS 2018 (pp. 641-6). Emerald Publishing Limited.

[52] Quaglio G, Dario C, Stafylas P, Tiik M, McCormack $\mathrm{S}$, Zilgalvis $\mathrm{P}$, et al. E-health in Europe: current situation and challenges ahead. Health Policy and Technology.2016; 5(4):314-7.

[53] Kanter AS, Borland R, Barasa M, Iiams-Hauser C, Velez $\mathrm{O}$, Nina Kaonga $\mathrm{N}$, et al. The importance of using open source technologies and common standards for interoperability within e-health: perspectives from the millennium villages project. In health information technology in the international context 2012 (pp. 189204). Emerald Group Publishing Limited. 
[54] Choudhury NR. Framework for development of information technology infrastructure for health (ITIH) care in india-a critical study. Qualitative and Quantitative Methods in Libraries. 2017; 5(4):787-96.

[55] Kavitha R, Kannan E, Kotteswaran S. Implementation of cloud based electronic health record (EHR) for Indian healthcare needs. Indian Journal of Science and Technology. 2016; 9(3):1-5.

[56] Kumar S, Kumar S, Mishra A, Yadav M. Developing a pro-people health policy: documenting the participatory processes involved in drafting and revision of national health policy. Journal of Health Management. 2017; 19(3):369-76.

[57] https://mohfw.gov.in/documents/policy. Accessed 13 March 2018.

[58] https://www.snomed.org/member/india. Accessed 13 March 2018.

[59] https://mohfw.gov.in/basicpage/electronic-healthrecord-ehr-standards-india-2016. Accessed 15 March 2018.

[60] Mudaly T, Moodley D, Pillay A, Seebregts CJ. Architectural frameworks for developing national health information systems in low and middle income countries. In proceedings of the first international conference on enterprise systems: ES 2013 (pp. 1-9). IEEE.

[61] Foster J. Case study 1: nursing informatics and ehealth in Australia. In introduction to nursing informatics 2015 (pp. 305-22). Springer, London.

[62] Khoshafian S. Service oriented enterprises. Auerbach Publications; 2016.

[63] Milosevic Z, Bond A. Digital health interoperability frameworks: use of RM-ODP standards. In 20th international enterprise distributed object computing workshop 2016 (pp. 1-10). IEEE.

[64] Walsh L, Hemsley B, Allan M, Adams N, Balandin S, Georgiou A, et al. The e-health literacy demands of Australia's my health record: a heuristic evaluation of usability. Perspectives in Health Information Management. 2017.

[65] Mather C, Cummings E, Gale F. Nurses as stakeholders in the adoption of mobile technology in Australian health care environments: interview study. JMIR Nursing. 2019; 2(1).

[66] Black AS. E-health-as-a-service: a service based design approach for large scale e-health architecture (Doctoral Dissertation, Queensland University of Technology).2018.

[67] Baskaya M, Yuksel M, Erturkmen GL, Cunningham M, Cunningham P. Health4Afrika-implementing HL7 FHIR based interoperability. In proceedings of world congress of medical and health informatics, MEDINFO. 2019:20-4.

[68] Huang F, Blaschke S, Lucas H. Beyond pilotitis: taking digital health interventions to the national level in China and Uganda. Globalization and Health. 2017; 13(49):1-11.

[69] Higman S, Dwivedi V, Nsaghurwe A, Busiga M, Sotter Rulagirwa H, Smith D, et al. Designing interoperable health information systems using enterprise architecture approach in resource-limited countries: a literature review. The International Journal of Health Planning and Management. 2019; 34(1): e85-99.

[70] Adebesin F, Kotzé P, Ritz D, Foster R, Van Greunen D. Method for selecting e-health standards to support interoperability of healthcare information systems. IST-Africa Conference Proceedings. 2014.

[71] Roa JH. Designing mobile instant messaging for collaborative health data management in Rwanda (Master's thesis). 2017.

[72] Jain S, Zorzi N. Investing for impact: the global fund approach to measurement of AIDS response. AIDS and Behavior. 2017; 21(1):91-100.

[73] Bholah LA, Beharee K. Mauritius e-health-trust in the healthcare revolution. In ITU kaleidoscope: trust in the information society (K-2015) 2015 (pp. 1-8). IEEE.

[74] Fox S, Duggan M. Mobile health 2010. Washington, DC: Pew Internet \& American Life Project; 2010.

[75] Cheerkoot-Jalim S, Gopal K. Design of an architectural framework for the implementation of ehealth in mauritius. In information and communication technology for sustainable development 2020 (pp. 547-58). Springer, Singapore.

[76] Katuu S. Health information systems, e-health strategy, and the management of health records: the quest to transform south africa's public health sector. In Healthcare Policy and Reform: Concepts, Methodologies, Tools, and Applications 2019:493517. IGI Global.

[77] http://policyresearch.limpopo.gov.za/bitstream/handle/ 123456789/873/e-Health\%20Strategy\%20South\% 20Africa.pdf? sequence=1. Accessed 13 March2018.

[78] Agostinho C, Ducq Y, Zacharewicz G, Sarraipa J, Lampathaki F, Poler R, et al. Towards a sustainable interoperability in networked enterprise information systems: trends of knowledge and model-driven technology. Computers in Industry. 2016; 79:64-76.

[79] Mgozi T, Weeks R. The impact of cloud computing on the transformation of healthcare system in South Africa. In ITU kaleidoscope: trust in the information society (K-2015) 2015 (pp. 1-7). IEEE.

[80] Njoroge M, Zurovac D, Ogara EA, Chuma J, Kirigia D. Assessing the feasibility of e-health and mHealth: a systematic review and analysis of initiatives implemented in Kenya. BMC Research Notes. 2017; 10(90):1-11.

[81] Kiberu VM, Mars M, Scott RE. Barriers and opportunities to implementation of sustainable ehealth programmes in Uganda: a literature review. African Journal of Primary Health Care \& Family Medicine. 2017; 9(1):1-10.

[82] Darcy N, Elias M, Swai A, Danford H, Rulagirwa H, Perera S. E-Health strategy development: a case study in Tanzania. Journal of Health Informatics in Africa. 2014; 2(2):36-43.

[83] https://www.who.int/goe/policies/countries/tza_ehealt h.pdf. 2012. Accessed 13 March 2018.

[84] Mashoka RJ, Murray B, George U, Lobue N, Mfinanga $J$, Sawe $\mathrm{H}$, et al. Implementation of 
electronic medical records at an emergency medicine department in Tanzania: the information technology perspective. African Journal of Emergency Medicine. 2019; 9(4):165-71.

[85] Chali F, Yonah ZO, Kalegele K. Data exchange architecture for the development of mobile applications that support e-health systems interoperability: a case of Tanzania. International Journal of Advanced Computer Research. 2018; $8(34): 1-10$

[86] https://www.healthcareitnews.com/news/interopera bility-3-charts-take-pulse-health-data-sharingtoday. Accessed 13 March 2018.

[87] Salas-Vega S, Haimann A, Mossialos E. Big data and health care: challenges and opportunities for coordinated policy development in the EU. Health Systems \& Reform. 2015; 1(4):285-300.

[88] Sukumar SR, Natarajan R, Ferrell RK. Quality of big data in health care. International Journal of Health Care Quality Assurance. 2015; 28(6):62134.

[89] Galetsi P, Katsaliaki K, Kumar S. Big data analytics in health sector: theoretical framework, techniques and prospects. International Journal of Information Management. 2020; 50:206-16.

[90] Goldstein BA, Navar AM, Pencina MJ, Ioannidis J. Opportunities and challenges in developing risk prediction models with electronic health records data: a systematic review. Journal of the American Medical Informatics Association. 2017; 24(1):198208.

[91] Cowie MR, Blomster JI, Curtis LH, Duclaux S, Ford I, Fritz F, et al. Electronic health records to facilitate clinical research. Clinical Research in Cardiology. 2017; 106(1):1-9.

[92] Union I. Communication from the commission to the European parliament, the council, the European economic and social committee and the committee of the regions. A New Skills Agenda for Europe. Brussels. 2014.

[93] Firouzi F, Farahani B, Ibrahim M, Chakrabarty K. Keynote paper: from EDA to IoT e-health: promises, challenges, and solutions. IEEE Transactions on Computer-Aided Design of Integrated Circuits and Systems. 2018; 37(12):2965-78.
[94] Eom D, Lee H. A holistic approach to exploring the divided standards landscape in e-health research. In ITU kaleidoscope: challenges for a data-driven society 2017 (pp. 1-7). IEEE.

[95] Payne JD. The state of standards and interoperability for m-health. Washington, DC: m-Health Alliance. 2013.

[96] Zeinab KA, Elmustafa SA. Internet of things applications, challenges and related future technologies. World Scientific News. 2017; 67(2):126-48.

[97] Agostinho C, Ducq Y, Zacharewicz G, Sarraipa J, Lampathaki F, Poler R, et al. Towards a sustainable interoperability in networked enterprise information systems: trends of knowledge and model-driven technology. Computers in Industry. 2016; 79:64-76.

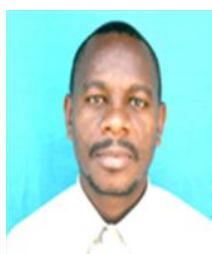

Mr. Marseline Michael Mtey is currently pursuing his $\mathrm{PhD}$ in Information and Communication Science and Engineering at NM-AIST. He completed his MSc in Computer Science at University of Dar es Salaam and BS Engineering from St Joseph University in Tanzania.

Email: mteym@nm-aist.ac.tz

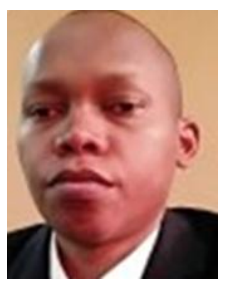

Dr. Mussa Ally Dida is currently working as a Lecturer at the Nelson Mandela African Institute of Science and Technology (NM-AIST) in Arusha, Tanzania. He obtained his $\mathrm{PhD}$ in Information and Communication Engineering from Beijing Institute of Technology in China, MSc in Telecommunication Engineering from the University of Dodoma and BSc in Computer Engineering and Information Technology from the University of Dar es Salaam both in Tanzania. 